\title{
BMJ Open Development of a binational thyroid cancer clinical quality registry: a protocol paper
}

\begin{abstract}
Liane J loannou, ${ }^{1}$ Jonathan Serpell, ${ }^{2,3}$ Joanne Dean, ${ }^{1}$ Cino Bendinelli, ${ }^{4}$ Jenny Gough, ${ }^{5}$ Dean Lisewski, ${ }^{6}$ Julie A Miller, ${ }^{7}$ Win Meyer-Rochow, ${ }^{8}$ Stan Sidhu, ${ }^{9}$ Duncan Topliss, ${ }^{10}$ David Walters, ${ }^{11}$ John Zalcberg, ${ }^{1}$ Susannah Ahern ${ }^{1}$
\end{abstract}

To cite: Ioannou LJ, Serpell J, Dean J, et al. Development of a binational thyroid cancer clinical quality registry: a protocol paper. BMJ Open 2019;9:e023723. doi:10.1136/ bmjopen-2018-023723

- Prepublication history and additional material for this paper are available online. To view these files, please visit the journal online (http://dx.doi. org/10.1136/bmjopen-2018023723).

Received 20 April 2018 Revised 19 October 2018 Accepted 19 0ctober 2018

Check for updates

(c) Author(s) (or their employer(s)) 2019. Re-use permitted under CC BY-NC. No commercial re-use. See rights and permissions. Published by BMJ.

For numbered affiliations see end of article.

Correspondence to

Dr Liane J loannou;

Liane.loannou@monash.edu

\section{ABSTRACT}

Introduction The occurrence of thyroid cancer is increasing throughout the developed world and since the 1990s has become the fastest increasing malignancy. In 2014, a total of 2693 Australians and 302 New Zealanders were diagnosed with thyroid cancer, with this number projected to rise to 3650 in 2018. The purpose of this protocol is to establish a binational population-based clinical quality registry with the aim of monitoring and improving the quality of care provided to patients diagnosed with thyroid cancer in Australia and New Zealand.

Methods and analysis The Australian and New Zealand Thyroid Cancer Registry (ANZTCR) aims to capture clinical data for all patients over the age of 16 years with thyroid cancer, confirmed by histopathology report, who have been diagnosed, assessed or treated at a contributing hospital. A multidisciplinary steering committee was formed which, with operational support from Monash University, established the ANZTCR in early 2017. The pilot phase of the registry is currently operating in Victoria, New South Wales, Queensland, Western Australia and South Australia, with over 20 sites expected to come on board across Australia in 2018. A modified Delphi process was undertaken to determine the clinical quality indicators to be reported by the registry, and a minimum data set was developed comprising information regarding thyroid cancer diagnosis, pathology, surgery and 90-day follow-up.

Future plans The establishment of the ANZTCR provides the opportunity for Australia and New Zealand to further understand current practice in the treatment of thyroid cancer and identify variation in outcomes. The engagement of endocrine surgeons in supporting this initiative is crucial. While the pilot registry has a focus on early clinical outcomes, it is anticipated that future collection of longer term outcome data particularly for patients with poor prognostic disease will add significant further value to the registry.

\section{INTRODUCTION}

Thyroid cancer is the fourth most common cancer in Australian males and third most common cancer in Australian females aged 15-39. ${ }^{1}$ The occurrence of thyroid cancer is increasing throughout the developed world, including Australia and New Zealand, and since

\section{Strengths and limitations of this study}

We outline the establishment of a binational clinical quality registry (CQR) for thyroid cancer, including the establishment of governance, recruitment framework, clinical quality indicators, minimum data set, data access policy and reporting structure. This CQR was developed as per the Australian Operating Principles for Clinical Quality Registries.

- There are very few established thyroid cancer registries internationally. This is a surgeon-driven optout CQR for thyroid cancer, with endocrine surgeons contributing data directly to the registry. This can be used as a model for researchers developing CQRs.

- Not all thyroid cancer surgery is performed by endocrine surgeons. Currently site participation, although desirable, does not require all surgeons performing surgery for thyroid cancer at a site to participate. This will be an important future activity for the registry.

- The time-consuming and labour-intensive site governance approval process in Australia and New Zealand is a major impediment for roll-out of the registry.

the 1990s, it has become the fastest increasing malignancy. Between 1991 and 2009, the number of thyroid cancer cases increased by $250 \%$ in Australia. ${ }^{2}$ In 2018, it is expected that there will be approximately 3300 new cases of thyroid cancer in Australia, ${ }^{3}$ and approximately 350 new cases in New Zealand. ${ }^{4}$ From 2007 to 2020 , thyroid cancer rates are projected to increase at a slightly lower rate by $33 \%$ in males and $62 \%$ in females. ${ }^{35}$

The most common types of thyroid cancer have very good long-term prognoses, and of all non-cutaneous cancers, thyroid cancer has the highest 5-year survival rate at 98\%. ${ }^{6}$ Patients with thyroid cancer typically undergo surgery with total thyroidectomy or hemithyroidectomy. After total thyroidectomy, higher risk patients may undergo thyroid bed ablation with radioactive iodine (RAI), followed by suppressive 
thyroxine therapy. The removal of the whole thyroid gland and RAI ablation results in a lifelong dependence on pharmacotherapy with thyroid hormone (levothyroxine). ${ }^{7}$

\section{Treatment complications}

Despite highly effective treatments and good long-term outcomes, a number of significant surgical and postoperative complications may occur and be associated with longterm physical and psychological morbidity. ${ }^{8}$ Specifically, complications of thyroidectomy, such as temporary voice change, may occur in up to $80 \%$ of patients, and permanent vocal cord palsy due to injury to the recurrent laryngeal nerve resulting in hoarseness, both potentially affect employment and quality of life. ${ }^{9-11}$ Postoperative hypocalcaemia, due to damage leading to inadequate functioning of remaining parathyroid glands (hypoparathyroidism), may cause symptoms such as severe cramps requiring prolonged inpatient stays on a temporary (in up to $15 \%$ ) or permanent (in $0.7 \%-3 \%$ ) basis following surgery ${ }^{12}$ and require permanent therapy with calcitriol therapy and calcium supplementation. Haemorrhage and wound infection are not uncommon, ${ }^{13}$ and there may be side effects from RAI treatment, such as xerostomia. Depending on cancer pathology and stage of disease, cancer recurrence may occur, requiring additional treatments. Distant metastases may occur in up to $6 \%-20 \%$ of cases at follow-up and markedly increase the risk of cancer-specific mortality. ${ }^{14}$

\section{Variation in management, treatment and outcome}

There are significant variations in the management, treatment and outcomes of thyroid cancer, particularly in the role of: diagnostic investigation and pretreatment scanning; optimal extent of surgery (total thyroidectomy or hemithyroidectomy); use of active surveillance for small low-risk cancers; central lymph node dissections (therapeutic and/ or prophylactic); outcomes following surgery (eg, recurrent laryngeal nerve palsy, hypoparathyroidism); postsurgical hormone treatment, calcium and vitamin D therapy; and RAI treatment (provision and dosage) ${ }^{15}$ Extent of surgery may be influenced by surgeon case volume (a measure of surgeon experience) and geographical location. ${ }^{16}$ Experienced surgeons are more likely to perform central neck dissections, arrange administration of RAI where appropriate and have lower rates of surgical complications. Thyroidectomies performed by high-volume thyroid surgeons have less than a $2 \%$ risk of hypoparathyroidism, recurrent laryngeal nerve injury and permanent paralysis (depending on the size of the primary tumour). In contrast, higher rates of complications occur when the procedure is performed by less experienced surgeons. ${ }^{17} 18$ It has therefore been recommended that surgeons operating on patients with thyroid cancer should perform a minimum of 20 thyroidectomies per year. ${ }^{19}$

\section{Clinical quality registries}

A proven strategy to reduce variation in outcomes is to measure and compare high-quality disease-specific data using clinical quality registries $(\mathrm{CQR})$. This strategy has been successfully tested in a range of surgical disciplines including trauma, ${ }^{20}$ cardiac surgery, ${ }^{21}$ transplantation, ${ }^{22}$ breast surgery, ${ }^{23}$ bariatric surgery, ${ }^{24}$ joint surgery ${ }^{25}$ and cancer care. ${ }^{26} \mathrm{CQRs}$ provide the most effective means of collecting high-quality data and are a tool for quality improvement. Where they have been introduced at a state or national level, CQRs have become one of the most clinically valued tools for quality improvement. ${ }^{27}$ The Australian Commission on Safety and Quality in Health Care (ACSQHC) has advocated development of CQRs, particularly in key high burden areas including cardiac disease, musculoskeletal disease and cancers. $^{28}$

\section{Measuring quality of care in thyroid cancer}

To date there are very few thyroid cancer registries internationally. Some of the more notable thyroid cancer registries include a prospective national clinical thyroid cancer database (DATHYRCA) implemented in 1996 by the Danish Head and Neck Cancer Group that collects data from the five Danish centres treating patients with thyroid carcinoma in Denmark; and the Thyroid Cancer Care Collaborative, a multi-institution thyroid cancer registry established in the USA in 1986 which includes 14 major academic medical centres and follows patients up annually to an average of 5 years. ${ }^{29}$ In the USA, the American Thyroid Association is collaborating with the Medullary Thyroid Carcinoma (MTC) Registry Consortium to establish a database of all patients newly diagnosed with MTC over the next $10-15$ years. While single-institution databases have been well published and provided extremely valuable data with regard to understanding thyroid cancer, little data have been published from multi-institution databases and/or registries regarding quality of thyroid cancer care. ${ }^{30}$

\section{Rationale}

Thyroid cancer management is informed by well-regarded international guidelines. ${ }^{31}$ However, given the lack of population-level data regarding patient outcomes from thyroid cancer in Australia and New Zealand, it is likely that there is clinician variation in adherence to best practice and therefore, individual patient outcomes of thyroid cancer. Furthermore, while detailed guidelines exist, there remain questions regarding optimal management of patient subpopulations. The Australian and New Zealand Thyroid Cancer Registry (ANZTCR) is a CQR being developed to provide a comprehensive evidence base regarding the care and outcomes of patients diagnosed with thyroid cancer in Australia and New Zealand. The registry will identify differences in quality of care and outcomes, with the aim to reduce variation and improve patient outcomes and survival. This paper describes the establishment and initial implementation of the ANZTCR.

\section{METHODS AND ANALYSIS}

\section{Establishment of ANZTCR}

In Australia, the majority of thyroid surgery is undertaken by specialist endocrine surgeons, represented by 
the Australian and New Zealand Endocrine Surgeons (ANZES). Long-standing and significant data regarding thyroid surgery have been collected at a number of academic and healthcare institutions across Australia. In 2016, ANZES agreed to lead the evolution of thyroid cancer quality improvement via the establishment of a multicentre, binational CQR for thyroid cancer which would include clinical indicators against which to monitor and benchmark clinical care. Across Australia and New Zealand it is likely that over 50 sites undertake surgery on patients with thyroid cancer including metropolitan, regional and private centres. Although confined to shortterm follow-up at the outset, the importance of obtaining patient follow-up information to provide further evidence regarding long-term outcomes following treatment for thyroid cancer has also been identified, and will be implemented in a subsequent phase of the registry.

\section{Patient and public involvement}

A patient and representative of the Australian Thyroid Foundation (ATF) is a member of the ANZTCR Steering Committee and was involved in the design of the study. Results will be disseminated to study participants via the ANZTCR website which will have links to all research output, reports and newsletters. The ATF will also play a role in dissemination of registry output to patients.

\section{Governance structure}

Coordinating centre

The ANZTCR Coordinating Centre is located in the Department of Epidemiology and Preventive Medicine at Monash University which manages the registry's core activities under the direction of the ANZTCR Steering Committee. Monash University has custodianship of the data which includes accountability for the privacy, security and integrity of patient information held within the registry.

\section{Site investigators}

The ANZTCR registry is a multicentred, investigator-driven endeavour. The primary investigator (PI) at each site is responsible for ensuring that research activities undertaken at their site are conducted in accordance with ethics committee approval, the research protocol, site registry agreements and related policy documentation. Site research activities include identification of patients for recruitment and data collection, overseen by the PI at each site.

\section{Steering committee}

The ANZTCR Steering Committee is multidisciplinary, chaired by the ANZTCR Clinical Lead and comprises representation of key stakeholders, including: endocrine surgeons (from each jurisdiction); endocrinologists; ANZES; consumers; database managers; and Monash University. The committe is also in the process of expanding to include an ear, nose and throat (ENT) surgeon representative. It meets quarterly and has a significant role in guiding registry strategy and policy, monitoring data collection and quality assurance, reviewing data requests and producing data reports and publications, as per the Australian Operating Principles for Clinical Quality Registries. ${ }^{32}$

\section{Management committee}

A management committee meets fortnightly and oversees the day-to-day running of the registry. Further subcommittees including a data access subcommittee will be established as required.

\section{Registry population}

All patients with a confirmed diagnosis of primary thyroid cancer $\geq 16$ years of age who have been diagnosed, assessed or treated at a participating site are eligible to participate in the registry.

\section{Opt-out process}

Recruitment to the registry uses an opt-out process which has been used successfully in over $75 \%$ of CQRs in Australia. ${ }^{33}$ The rationale for this approach is based on minimising selection bias by achieving near $100 \%$ coverage of a population. By limiting the possibility of 'cherry picking' participants or omitting specific groups of patients otherwise not able to be captured by standard consenting processes, clinical validity increases, enabling meaningful analysis and comparison of variation in health outcomes across sites and other geographical areas. The opt-out process enables the full spectrum of public health information to be reported and analysed, increasing capacity to influence and inform clinical guidelines, policy development and funding decisions. ${ }^{34-36}$

\section{Participant recruitment}

Patient recruitment at a participating site commences following the appointment of a PI to take responsibility for the registry at the site, and authorisation by the participating site's research governance office. An outline of the recruitment framework is provided in figure 1.

Phase 1: All patients diagnosed with thyroid cancer, based on histological confirmation (provided approximately 1-2 weeks after surgery) at a hospital with ANZTCR research governance approval are eligible to participate. The treating endocrine surgeon (or designated staff member) at the surgical endocrine unit will enter minimal patient details into the ANZTCR Research Electronic Data Capture (REDCap) database, in addition to confirming thyroid cancer diagnosis and patient disclosure, at sites where a waiver of consent has been approved for the clinician to provide this information to the registry.

Phase 2: The Monash University ANZTCR coordinating centre will identify patients in the registry and invite them to participate in the study via a mail-out. The mail-out will include an introductory letter explaining the study, including information about the purpose, and possible outcomes of the research (including publication of research results) and a copy of the ANZTCR Participant Explanatory Statement. Using the opt-out process 


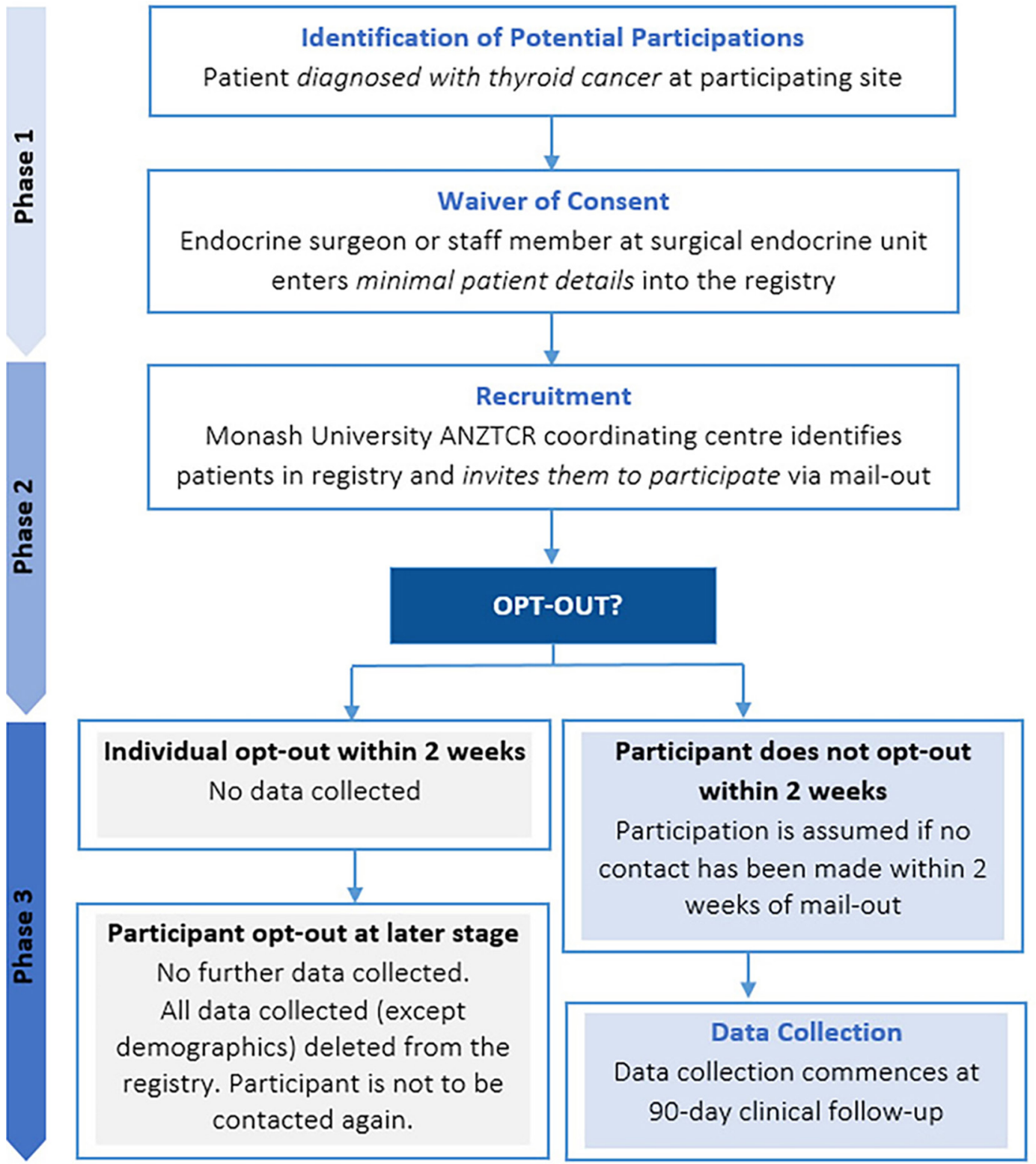

Figure 1 Patient recruitment framework. ANZTCR, Australian and New Zealand Thyroid Cancer Registry.

the patient will contact Monash University if they choose to not participate in the study. If the patient does not contact the study coordinator within two weeks participation is assumed.

Phase 3: If the participant has not opted out of the registry the endocrine surgeon will enter participant diagnosis, surgical, pathology and treatment data into the registry database 90 days after surgery.

\section{Clinician engagement}

The ANZTCR is an observational study of patients with newly diagnosed thyroid cancer receiving surgical treatment. It collects identifiable patient key diagnostic, clinical, treatment and complication data from diagnosis to 90 days post-thyroid cancer surgery. The registry is multicentred operating across participating sites in Victoria, New South Wales, Queensland, Western Australia and South Australia, and expanding to New Zealand in 2019.
Since the establishment of the registry we have received interest from almost 30 endocrine surgeons from over 35 sites throughout Australia and New Zealand.

Endocrine surgeons are informed about the registry through ANZES. Quarterly ANZTCR newsletters are distributed to all ANZES members to inform them of the activities and progress of the registry. Registry staff visit clinicians at their participating hospitals to introduce the registry. The PI and the associate investigator for the registry at each site also act as ambassadors for the registry and promote participation within their site. Clinician participation forms are sent to all endocrine surgeons, outlining the project and inviting clinicians to participate in the registry.

The Monash University ANZTCR coordination centre is responsible for distributing the clinician participation forms to surgeons and collating signed forms prior to 
the recruitment of patients and data collection. This is a once-only process for clinicians and provides agreement by the surgeon to participate in the registry and enter data on all patients for whom they are listed as the diagnosing or treating clinician in participating hospitals and private practice.

\section{Data collection}

Patient demographic and clinical data are submitted by sites primarily via direct data entry using REDCap, a secure web-based database. Clinicians (or their data managers) are responsible for entering their patient data directly into the ANZTCR REDCap database (ANZTCR-RCD). Opportunities to import data into the ANZTCR-RCD from existing institutional databases are also being developed. Site staff are trained to use the database and are provided with a data entry manual to assist with good quality data collection. Data to be collected include demographic and clinical data up to 90 days' follow-up. ANZTCR staff will check case ascertainment periodically via each site's Health Information Services (HIS) unit. The use of agreed definitions of the data elements ensures that the information collected is consistent and uniform, providing reliable and comparable data for analysis. A detailed data dictionary containing the data elements, formats, ranges and validation rules and definitions has been developed and will be maintained under document management with version control.

\section{Minimum data set and quality indicators}

To benchmark clinical care, CQRs require systematic measurement at predefined intervals and the capacity to report back information to participating clinical units. A modified Delphi approach, informed by international Thyroid Cancer Guidelines and relevant literature, was used to develop a set of thyroid cancer clinical indicators, the parameters of which are shown in table 1. A detailed methodology of the modified Delphi process will be published separately. Following the development of the clinical indicators, a minimum data set was developed that included variables relating to the indicators, variables required for patient identification and contact, and other variables of particular relevance to early thyroid cancer management. The selection of data fields and their definitions were derived from national data specifications such as Metadata Online Registry where they exist and from international thyroid cancer registry data dictionaries where terms are not defined within the Australian context. Once a final list was generated it was then endorsed by the ANZTCR Steering Committee. Data items collected by the ANZTCR are outlined in table 2.

\section{Data access policy}

Clinicians can access their own data through the registry database. Researchers may access registry data following approval by the ANZTCR Steering Committee and
Table 1 Framework of consensus set of clinical quality indicators

\begin{tabular}{|c|c|c|}
\hline Reference No & Clinical quality indicator & \\
\hline \multicolumn{3}{|l|}{ Preoperative } \\
\hline CQI 1 & Ultrasound (US) & Process \\
\hline CQI 2 & Fine needle aspiration (FNA) & Process \\
\hline CQI 3 & Voice assessment & Process \\
\hline \multicolumn{3}{|l|}{ Surgery } \\
\hline CQI 4 & Extent of surgery & Process \\
\hline CQI 5 & Lymph node dissection & Process \\
\hline \multicolumn{3}{|c|}{ Surgical complications } \\
\hline CQI 6 & $\begin{array}{l}\text { Recurrent laryngeal nerve } \\
(\mathrm{RLN}) \text { palsy }\end{array}$ & Outcome \\
\hline CQI 7 & $\begin{array}{l}\text { Hypoparathyroidism } \\
\text { (hypocalcaemia) }\end{array}$ & Outcome \\
\hline CQI 8 & $\begin{array}{l}\text { Haemorrhage within } 48 \text { hours } \\
\text { (requiring return to theatre) }\end{array}$ & Outcome \\
\hline \multicolumn{3}{|c|}{ Staging and treatment planning } \\
\hline CQI 9 & Postoperative TNM staging & Process \\
\hline CQI 10 & $\begin{array}{l}\text { Multidisciplinary team meeting } \\
\text { (MDM) }\end{array}$ & Process \\
\hline \multicolumn{3}{|c|}{ Postsurgical treatment } \\
\hline CQI 11 & Completion thyroidectomy & Process \\
\hline CQI 12 & Serum thyroglobulin $(\mathrm{Tg})$ & Process \\
\hline CQI 13 & Radioactive iodine (RAI) & Process \\
\hline
\end{tabular}

CQI, clinical quality indicator; TNM, tumor, node, metastases.

Ethics Committee as per the ANZTCR Data Access Policy (online supplementary material). ANZTCR Coordinating Centre, based at Monash University, is the point of contact for matters relating to access to registry data.

\section{Reporting}

As per the 2008 ACSQHC Operating Principles, the ANZTCR will undergo a period of establishment and on-boarding of participating sites before the commencement of regular reporting. At the conclusion of approximately two years, the ANZTCR will be reviewed regarding its achievement against its aims and suitability for further roll-out. Beyond twoyears and when sufficiently mature data are available, the ANZTCR is anticipated to produce a range of regular reports including: annual reports and benchmarked deidentified reports of clinical quality indicators (with identified data made available confidentially to participating sites).

\section{DISCUSSION}

Following its national establishment, the ANZTCR will monitor diagnosis, treatment and outcomes allowing for the identification of patterns of care and practices associated with better outcomes through improved compliance with best practice-based guidelines for the management 


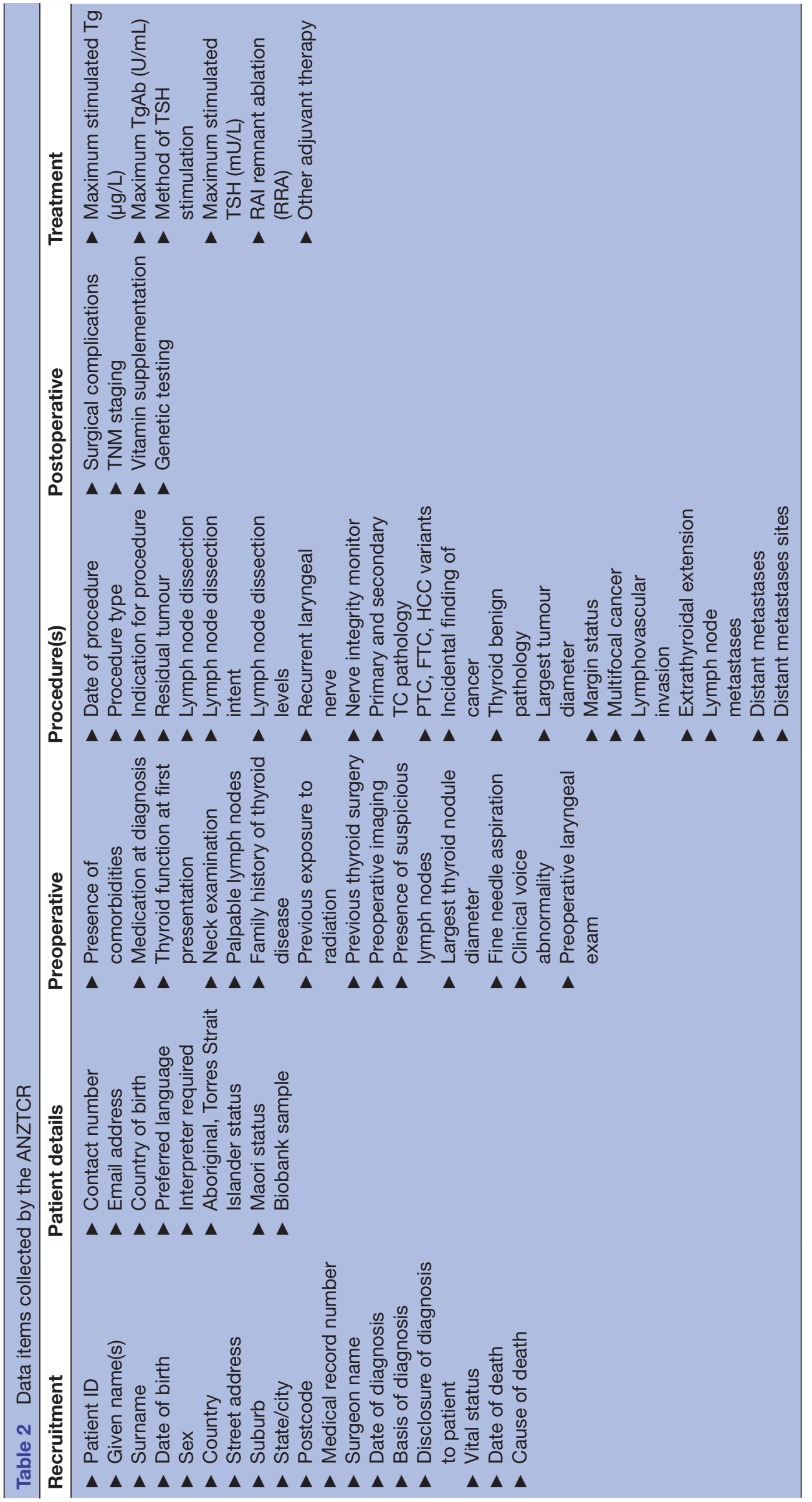


of thyroid cancer. The registry will also be able to identify risk factors that predict favourable and unfavourable treatment outcomes, postoperative complications and prognosis, leading to the stratification of treatments and follow-up. The ANZTCR will highlight risks and benefits of specific approaches to thyroid cancer as well as establish international benchmarks in the routine management of thyroid cancer surgical care. Further, the ANZTCR provides a basis as a platform for longer term clinical follow-up, substudies exploring treatment outcomes and clinical trials. We believe that the ANZTCR through its accumulation of a significant thyroid cancer cohort will assist in identifying best practice management specifically in complex poor prognostic thyroid cancer cases of low incidence.

One of the key features of the ANZTCR is that it is a surgeon-based CQR, with surgeons entering their patient data directly into the ANZTCR-RCD. While there are a number of benefits to this structure, including reduced data collection costs related to hiring additional staff and subject-specific training, there are also a number of potential challenges. One of the major challenges is surgeon engagement. However, due to the registry being supported by the ANZES, a specialty society of endocrine surgeons, we have had high engagement during the establishment phase. In order to maintain this engagement we have circulated quarterly newsletters with updates on the registry and invited the surgeons to be involved in the development of the clinical quality indicators and minimum data set. Continual review and refinement of the data set will ensure that the data collection burden is kept to a minimum. Additionally, the registry database allows surgeons to run site patient-level and aggregate data reports in real time for use in clinical care. The registry will also provide surgeons with an 'ANZTCR Valued Contributor' logo for use on their email signature, letterhead and/or website, and has been approved as a clinical audit activity by the Royal Australian College of Surgeons Continuing Professional Development programme.

Data from CQRs generally have strong external validity particularly with regard to generalisability and extrapolation of outcomes, however bias can exist, ${ }^{37}$ particularly due to the nature of the registry data being surgeon derived. Initial quality assurance processes will include sample audits of participating surgeon records. In the future to ensure case ascertainment, the ANZTCR could potentially receive monthly data extracts from participating site HIS or the Victorian Cancer Registry, pending approval. As the registry expands it will also engage non-specialist endocrine surgeons who undertake thyroid cancer surgery including ENT surgeons.

In order to commence patient recruitment the registry needs to seek ethics approval and governance authorisation at the participating site. As CQRs are becoming more common, standard guidelines have been introduced to make the ethics process more manageable. In particular, the National Mutual Acceptance (NMA) scheme has streamlined ethics for all public hospitals in all states except Tasmania (and the Northern Territory). Private hospitals can still choose to participate through the NMA scheme. Nevertheless, the process of obtaining site governance approval remains both time consuming and labour intensive. ${ }^{38}{ }^{39}$ Finally, perhaps the most important challenge faced by CQRs includes ongoing funding to ensure their sustainability. Currently the ANZTCR has enough funding to support the registry during the initial pilot phase, however in order to progress to national roll-out and implement longer term clinical follow-up and patient-reported outcomes additional funding is required. This is particularly relevant as lengthy follow-up is required to ascertain outcomes relating to recurrence. ${ }^{30} 40$ Nevertheless, given the benefits to patients, clinicians and wider stakeholders, and high level of clinician engagement, we are optimistic that in time these benefits will be realised.

\section{Future directions}

The registry is currently in a pilot phase to assess feasibility and clinician acceptability. The long-term aims of the registry, following conclusion of the pilot and dependant on funding, are to include longer term follow-up data from patients, multidisciplinary clinicians and data linkage, with a focus on cancer survivorship issues and the management of poorer prognostic and recurrent cancers.

\section{Author affiliations}

${ }^{1}$ Department of Epidemiology and Preventive Medicine, Monash University,

Melbourne, Victoria, Australia

${ }^{2}$ Endocrine Surgery Unit, Alfred Hospital, Melbourne, Victoria, Australia

${ }^{3}$ Endocrine Surgery Unit, Monash University, Melbourne, Victoria, Australia

${ }^{4}$ Department of Surgery, John Hunter Hospital, New Lambton Heights, New South Wales, Australia

${ }^{5}$ Breast and Endocrine Surgery, The Wesley Hospital, Queensland, Australia

${ }^{6}$ Department of General Surgery, Fiona Stanley Hospital, Perth, Western Australia, Australia

${ }^{7}$ Endocrine Surgery Unit, The Royal Melbourne Hospital, Melbourne, Victoria, Australia

${ }^{8}$ Department of Surgery, Waikato Hospital, Hamilton, New Zealand

${ }^{9}$ Endocrine Surgery Unit, University of Sydney, Royal North Shore Hospital, Sydney, New South Wales, Australia

${ }^{10}$ Department of Endocrinology and Diabetes, Alfred Health, Melbourne, Victoria, Australia

${ }^{11}$ Breast and Endocrine Surgical Unit, University of Adelaide, The Queen Elizabeth Hospital, Sydney, New South Wales, Australia

Acknowledgements The authors gratefully acknowledge the Australian and New Zealand Endocrine Surgeons (ANZES) and the Australian Thyroid Foundation (ATF) for their ongoing support of the ANZTCR. We also acknowledge the contribution of Ms Madeleine Allnutt, representative of the Australian Thyroid Foundation and member of the ANZTCR Steering Committee.

Collaborators Australian and New Zealand Endocrine Surgeons (ANZES) and the Australian Thyroid Foundation (ATF).

Contributors LJI, JD and SA wrote the first draft of the manuscript and developed the registry protocol. JS has led the development of the Australian and New

Zealand Thyroid Cancer Registry (ANZTCR) and is the chair of the ANZTCR Steering Committee. The remaining authors, CB, JG, DL, JAM, WMR, SS, DT, DW and JZ, are members of the steering committee, were involved in the protocol development, reviewed and provided feedback on various drafts of the manuscript, and approved the final manuscript.

Funding The authors gratefully acknowledge the Alfred Foundation and their industry partner, Medtronic, for their funding of the ANZTCR. 
Competing interests None declared.

Patient consent Not required.

Ethics approval This project has received ethics approval from Alfred Health HREC under the National Mutual Acceptance (NMA) scheme (HREC/16/Alfred/61).

Provenance and peer review Not commissioned; externally peer reviewed.

Open access This is an open access article distributed in accordance with the Creative Commons Attribution Non Commercial (CC BY-NC 4.0) license, which permits others to distribute, remix, adapt, build upon this work non-commercially, and license their derivative works on different terms, provided the original work is properly cited, appropriate credit is given, any changes made indicated, and the use is non-commercial. See: http://creativecommons.org/licenses/by-nc/4.0/.

\section{REFERENCES}

1. Western Australian Cancer Registry. Cancer incidence and mortality in Western Australia. Data Integrity directorate PaSPD. editor. Perth Western Australia: Department of Health, 2014.

2. Australian Government. Cancer in Australia: an overview. 2012Australian Institute of Health and Welfare (AlHW), Australasian Association of Cancer Registries. editors. Canberra: AlHW, 2012.

3. Australian Government. Australian Cancer Database (ACD). Australian Institute of Health and Welfare (AlHW), Australasian Association of Cancer Registries. editors. Canberra: AlHW, 2011.

4. Cancer Data and Stats. Mo H, ed. New Zealand Cancer Registry (NZCR). Wellington: New Zealand Government, 2017.

5. Australian Government. National Mortality Database (NMD). Australian Institute of Health and Welfare (AlHW), Australasian Association of Cancer Registries. editors. Canberra: AlHW, 2011.

6. Jemal A, Ward EM, Johnson CJ, et al. Annual Report to the Nation on the Status of Cancer, 1975-2014, featuring survival. J Natl Cancer Inst 2017;109.

7. Husson O, Haak HR, Mols F, et al. Development of a disease-specific health-related quality of life questionnaire (THYCA-QoL) for thyroid cancer survivors. Acta Oncol 2013;52:447-54.

8. Mendoza A, Shaffer B, Karakla D, et al. Quality of life with welldifferentiated thyroid cancer: treatment toxicities and their reduction. Thyroid 2004;14:133-40.

9. Lee JC, Breen D, Scott A, et al. Quantitative study of voice dysfunction after thyroidectomy. Surgery 2016;160:1576-81.

10. Serpell JW, Lee JC, Yeung MJ, et al. Differential recurrent laryngeal nerve palsy rates after thyroidectomy. Surgery 2014;156:1157-66.

11. Hayward NJ, Grodski S, Yeung M, et al. Recurrent laryngeal nerve injury in thyroid surgery: a review. ANZ J Surg 2013;83:15-21.

12. Serpell JW. Preventing hypoparathyroidism after total thyroidectomy. ANZ J Surg 2018;88:127-8.

13. Lee JC, Chang P, Grodski S, et al. Temporal analysis of thyroid cancer management in a Melbourne tertiary centre. ANZ J Surg 2016;14.

14. Nixon IJ, Whitcher MM, Palmer FL, et al. The impact of distant metastases at presentation on prognosis in patients with differentiated carcinoma of the thyroid gland. Thyroid 2012;22:884-9.

15. Hall SF, Irish JC, Groome PA, et al. Practice patterns in the management of patients with differentiated thyroid cancer in Ontario Canada 2000-2008. J Otolaryngol Head Neck Surg 2014;43:29.

16. Tasevski R. Management patterns and outcomes of differentiated thyroid cancer in ontario: a population-based study. (Doctoral dissertation). 2013.

17. Cobin $\mathrm{RH}$, Gharib H, Bergman DA, et al. AACE/AAES medical/ surgical guidelines for clinical practice: management of thyroid carcinoma. American association of clinical endocrinologists. american college of endocrinology. Endocr Pract 2001;7:202.

18. Pacini F, Schlumberger M, Dralle H, et al. European consensus for the management of patients with differentiated thyroid carcinoma of the follicular epithelium. Eur J Endocrinol 2006;154:787-803.
19. Perros P, Boelaert K, Colley S, et al. British thyroid association guidelines for the management of thyroid cancer. Third Edition. Clin Endocrinol 2014;81:1-122.

20. Cameron PA, Gabbe BJ, McNeil JJ, et al. The trauma registry as a statewide quality improvement tool. J Trauma 2005;59:1469-76.

21. Victorian Cardiac Surgery Database Project. Australasian society of cardiac and thoracic surgeons database project steering committee: Victorian Cardiac Surgery Database Project.

22. Australia and New Zealand Dialysis and Transplant Association (ANZDATA). Australia and new zealand dialysis and transplant registry [Web Page]. http://www.anzdata.org.au/

23. Malycha P, Tyson S. National breast surgery audit. Aust N Z J Surg 2000;70:834-6.

24. Poelemeijer YQM, Liem RSL, Nienhuijs SW. A Dutch Nationwide Bariatric Quality Registry: DATO. Obes Surg 2018;28:1602-10.

25. Graves SE, Davidson D, Ingerson L, et al. The Australian Orthopaedic Association National Joint Replacement Registry. Med J Aust 2004;180:S31.

26. Cancer Institute NSW. NSW clinical cancer registry [Web Page]. http://www.cancerinstitute.org.au/cancer_inst/programs/registryccr. html

27. Sweden E. Handbook for establishing quality registries. Karlskrona: Eyenet Sweden, 2005

28. ACSQHC. Australian commission on safety and quality in health care. information strategy. Sydney: ACSQHC, 2007.

29. Londero SC, Mathiesen JS, Krogdahl A, et al. Completeness and validity in a national clinical thyroid cancer database: DATHYRCA. Cancer Epidemiol 2014;38:633-7.

30. Mehra S, Tuttle RM, Milas M, et al. Database and registry research in thyroid cancer: striving for a new and improved national thyroid cancer database. Thyroid 2015;25:157-68.

31. Haugen BR, Alexander EK, Bible KC, et al. 2015 American Thyroid Association Management Guidelines for Adult Patients with Thyroid Nodules and Differentiated Thyroid Cancer: The American Thyroid Association Guidelines Task Force on Thyroid Nodules and Differentiated Thyroid Cancer. Thyroid 2016;26:1-133.

32. Australian Commission on Safety and Quality in Health Care. Operating Principles and Technical Standards for Australian Clinical Quality Registries [Web Page]. http://www.safetyandquality.gov.au/ internet/safety/publishing.nsf/Content/EA520128CB313DE9CA25 73AF007BC590/\$File/OP TS-Nov2008.pdf.

33. Tu JV, Willison DJ, Silver FL, et al. Impracticability of informed consent in the Registry of the Canadian Stroke Network. N Engl J Med 2004;350:1414-21.

34. Cancer Council of Victoria. Cancer Council of Victoria Registry and Statistics. 2013 http://www.cancervic.org.au/research/registrystatistics

35. Junghans $\mathrm{C}$, Jones $\mathrm{M}$. Consent bias in research: how to avoid it. Heart 2007;93:1024-5.

36. Buckley B, Murphy AW, Byrne M, et al. Selection bias resulting from the requirement for prior consent in observational research: a community cohort of people with ischaemic heart disease. Heart 2007;93:1116-20.

37. Stub D, Lefkovits J, Brennan AL, et al. The Establishment of the Victorian Cardiac Outcomes Registry (VCOR): Monitoring and Optimising Outcomes for Cardiac Patients in Victoria. Heart Lung Circ 2018;27:451-63.

38. Clay-Williams R, Taylor N, Braithwaite J. Potential solutions to improve the governance of multicentre health services research. Med J Aust 2018;208:152-4.

39. Brown WA, Smith BR, Boglis M, et al. Streamlining ethics review for multisite quality and safety initiatives: national bariatric surgery registry experience. Med J Aust 2016;205:200-1.

40. Pearce EN, Lee SL, Weiss R, et al. Unique obstacles to establishing thyroid cancer registries. J Clin Trans/ Endocrinol 2016;3:12-13. 